\title{
Processamento Visual da Forma em Humanos: Curvas de Limiar de Contraste para Padrões Circularmente Simétricos
}

\author{
Natanael Antonio dos Santos ${ }^{12}$ \\ Universidade Federal da Paraíba \\ Maria Lúcia de Bustamante Simas \\ Renata Maria Toscano Barreto Lyra Nogueira \\ Universidade Federal de Pernambuco
}

\begin{abstract}
Resumo
O objetivo deste trabalho foi mensurar curvas de limiar de contraste para padrões espaciais circularmente simétricos em coordenadas polares. Mensurou-se os limiares de contraste para grade senoidais, freqüências radiais e freqüências radiais acopladas à freqüência angular de 4 ciclos $\backslash 360^{\circ}$. Participaram dos experimentos 6 adultos jovens com acuidade visual normal ou corrigida. As freqüências estimadas com o paradigma psicofísico da escolha forçada foram: 0,$2 ; 0,3 ; 0,5 ; 0,8 ; 1 ; 2 ; 3 ; 4 ; 6$ e 9 cpg. Os resultados mostraram que o acoplamento de freqüências radiais à freqüência angular causou um aumento na sensibilidade do sistema visual humano de pelo menos 1,6 em relação à curva de sensibilidade ao contraste para estímulos radiais puros. Estes resultados são consistentes com o modelo de canais múltiplos de freqüências espaciais para estímulos definido a priori em termos de coordenas polares.

Palavras-chave: Processamento visual da forma; freqüência angular; freqüência radial; sensibilidade ao contraste; método da escolha forçada.
\end{abstract}

Visual Processing of Form in Human: Threshold Contrast Curves to Concentrically Symmetric Patterns

\begin{abstract}
The aim of this work was to measure contrast sensitivity curves to concentrically symmetric patterns in a system of polar coordinates. We measured the contrast thresholds to sine wave gratings, radial frequencies and coupled radial/angular frequencies. Six young adults with corrected-to-normal visual acuity participated in the experiments. The frequencies measured with a forced-choice psychophysical method were $0.2,0.3,0.5,0.8,1.0,2.0,3.0,4.0,5.0,6.0$ and $9.0 \mathrm{cpd}$. Results showed that coupled radial/angular frequencies caused an increase in the sensitivity of at least 1.6 times in relation to radial contrast sensitivity function. These results were in agreement with the model of multiple channels processing of spatial frequencies of stimuli defined in terms of polar coordinates.

Keywords: Visual processing of form; radial frequency; angular frequency; contrast sensitivity; forced choice method.
\end{abstract}

Campbell e Robson (1968) propuseram o modelo de canais múltiplos para estudar o processamento visual da forma e contraste. De acordo com Campbell e Robson, o sistema visual humano (SVH) responde seletivamente a freqüências espaciais, ou seja, o sistema visual (SV) processa simultaneamente diferentes faixas estreitas de freqüências espaciais (Blakemore \& Campbell, 1969b; Campbell \& Robson, 1968; Sachs, Nachmias \& Robson, 1971). Em outras palavras, a abordagem de canais múltiplos ou análise de sistema linear defende que a percepção de um objeto qualquer ocorre porque o nosso SV executa uma análise ou uma decomposição desse padrão complexo em seus componentes mais elementares, uma espécie de análise de Fourier. Santos e Simas (2001a) discutem boa parte das evidências psicofísicas e neurofisiológicas favoráveis a abordagem de canais múltiplos de freqüências espaciais.

\footnotetext{
${ }^{1}$ Suporte Financeiro: CNPq.

${ }^{2}$ Endereço para correspondência: Departamento de Psicologia, CCHLA UFPB. Cidade Universitária, 58051 900, João Pessoa, PB. Fone: (83) 2354439 , Fax (83)2167064.E-mail: natanael@cchla.ufpb.br
}

O modelo de canais múltiplos assume que a curva de limiar de contraste (1/CSF) ou a função de sensibilidade ao contraste (CSF) é o envelope da sensibilidade para a série total de canais, cada um sensível a uma região do espectro (Braddick, Campbell \& Atkinson, 1978; Graham \& Nachmias, 1971; R. L. De Valois \& K. K. De Valois, 1988). Neste contexto, a CSF tem sido utilizada para descrever e compreender os mecanismos putativos que medeiam o processamento visual da forma e de detalhes espaciais. Limiar de contraste pode ser definido como a quantidade mínima de contraste necessária para detectar um padrão qualquer (Ex.: uma grade) de uma determinada freqüência espacial (Cornsweet, 1970). Assim, o contraste para cada freqüência espacial é ajustado com um procedimento comportamental ou psicofísico até que o SV possa descriminar um padrão (ou objeto) de freqüência espacial de um campo homogêneo de luminância média. Em resumo, a CSF estima a visibilidade de qualquer objeto em função de sua freqüência espacial.

Atualmente, estudos psicofísicos em humanos e estudos neurofisiológicos em macacos e gatos fornecem forte suporte para a idéia de que o processamento visual de objetos é 
composto por mecanismos ou canais sintonizados para bandas estreitas de freqüências espaciais (R. L. De Valois \& K. K. De Valois, 1988; Santos \& Simas, 2001a; Simas \& Santos, 2002a; Wilson, Levi, Maffei, Rovamo \& De Valois, 1990).

\section{A Estimulação Visual: Estímulos Elementares}

$\mathrm{Na}$ investigação da percepção ou do processamento visual da forma e de contraste dentro da perspectiva de canais múltiplos, as características matemáticas e físicas que definem o estímulo visual no espaço são fundamentais, principalmente, a medida em que estas possam ser relacionadas à detecção e ao reconhecimento ou ao processamento neural que envolve a análise e síntese da imagem percebida. Neste sentido, a variação dos níveis de luminância que determinam o contraste (ou a luminância média que determina o brilho perceptivo) e a freqüência espacial assumem um papel importante na percepção visual da forma. Assim, a mensuração da sensibilidade ao contraste em função da configuração espacial que o estímulo pode assumir é um dos aspectos essenciais para se compreender e modelar mecanismos envolvidos no processamento visual de objetos.

\section{Padrão Espacial Circularmente Simétrico e a Função de Sensibilidade ao Contraste}

Os estudos clássicos que procuraram caracterizar os mecanismos responsáveis pelo processamento de contraste no SVH se basearam principalmente em resultados com grades senoidais verticais (Blakemore \& Campbell, 1969a, 1969b; Blakemore, Nachmias \& Sutton, 1970; Campbell \& Maffei, 1970, 1974; Campbell \& Robson, 1968; Graham, 1972; Maffei \& Fiorentini, 1973; Pantle \& Sekuler, 1968), estímulos cartesianos semelhantes aos encontrados na Figura 1. Nas últimas décadas, alguns pesquisadores procuraram estabelecer a existência de canais múltiplos para padrões espaciais diferentes de grade senoidal, por exemplo, freqüências angulares (Santos \& Simas, 2002; Simas, 1985; Simas \& Dodwell, 1990; Simas, Frutuoso \& Vieira, 1992; Simas \& Santos, 2002a, 2002b). Entretanto, a primeira proposta de estudar padrões em coordenadas polares ao invés de coordenadas cartesianas (grade senoidal) partiu de Kelly (1960), que sugeriu estímulos circularmente simétricos, os quais ele denominou de alvo $\mathrm{J}_{0}$, isto é, estímulos modulados pela função cilíndrica de Bessel de ordem zero. Esta classe de padrões tem recebido outras denominações, como por exemplo, "alvo" (Bull's-eye), grades circulares, dentre outras. Nós temos denominado de estímulo elementar de freqüência radial porque a modulação de contraste varia senoidalmente ou cossenoidalmente na direção radial, considerando o centro do círculo como a origem do sistema de coordenadas polares (Figura 1). Kelly discutiu que padrões deste tipo poderiam ser mais apropriados para estudar o SV considerando a simetria aproximadamente circular da retina.
Apesar de Kelly ter proposto estímulo dessa natureza desde 1960, o mesmo só foi utilizado 15 anos depois, quando Kelly e Magnuski (1975) utilizaram padrões de freqüências radiais circularmente simétricos para estimar a CSF do SVH. De acordo com estes autores, padrões modulados pela função cilíndrica de Bessel parecem ser mais naturais como estímulos visuais, fornecendo um centro claro de fixação. O mesmo não acontece com padrões em coordenadas cartesianas, por exemplo, grade senoidal (Figura 1). O principal objetivo de Kelly e Magnuski foi comparar a CSF estimada para grades senoidais com a FSC estimada para freqüências radiais moduladas pela função cilíndrica de Bessel, $\mathrm{J}_{0}$. $\mathrm{O}$ fato é que nenhum outro artigo aparece na literatura até 1982, quando Kelly replicou parte do estudo de 1975, com a técnica psicofísica de ajustamento de contraste, onde o voluntário ajustava seu próprio limiar de contraste. Os resultados obtidos por Kelly demonstraram que a faixa de maior sensibilidade para $\mathrm{J}_{0}$ foi por volta de 0,5-2,0 cpg. Os resultados de Kelly também mostraram que o SVH é mais sensível para estímulos do tipo grade senoidal do que para estímulos radiais.

Simas e Santos (2002b)mediram a sensibilidade ao contraste para estímulos radiais modulados pela função esféricade Bessel para vários jn $(n=0 ; 1 ; 2 ; 4 ; 8 ; 16)$ com o paradigma psicofísico da escolha forçada. $\mathrm{O} n$ representa a ordem da função esférica de Bessel. Os resultados demonstraram que a faixa de máxima sensibilidade do SVH para $j_{0}$ se deu por volta de $1 \mathrm{cpg}$, enquanto que para $j_{16}$ se deu por volta de 2 e $3 \mathrm{cpg}$.

Atualmente podemos encontrar na literatura outros trabalhos com estímulos circularmente simétricos (Ex.: Amidor, 1997; Gallant, Brau \& van Essen, 1993; Gallant, Connor, Rakshit, Lewis \& van Essen 1996; Hegde \& Van Essen, 2000; Hess, Wang, Demanins, Wilkinson \& Wilson, 1999; Mortensen \& Meinhardt, 2001; Verrall \& Kakarala, 1998; Wilkinson, Wilson, \& Habak, 1998; Wilson \& Wilkinson, 1997, 1998; Wilson, Wilkinson \& Asaad, 1997), entretanto, estes utilizaram sugestões teóricas diferentes de Kelly (Kelly, 1960, 1982; Kelly \& Magnuski, 1975), e Simas (Simas, 1985; Simas \& Dodwell, 1990; Simas \& Santos 2002a, 2002b). Por exemplo, os estudos de Gallant e colaboradores $(1993,1996)$ utilizaram estímulos com modulação de onda senoidal ou quadrada baseados em freqüências radiais e angulares para testar a resposta de células na área visual V4 de macacos.

\section{O Presente Estudo}

Este estudo dá continuidade aos experimentos que procuram caracterizar mecanismos ou respostas típicas do SVH para padrões de freqüências espaciais circularmente simétricos em coordenadas polares. Boa parte destes 
experimentos acreditam na possibilidade das áreas superiores do SV (Ex.: área visual V4 e córtex ínfero-temporal, IT) processarem informação visual da forma em termos de freqüências radiais e angulares, o que pode envolver filtragem de faixas ou áreas inteiras do campo visual. No presente estudo, mensuramos a CSF para grades senoidais, freqüências radiais e freqüências radiais/angulares acopladas moduladas pela função cilíndrica de Bessel, $\mathrm{J}_{0}$. O objetivo principal foi caracterizar a resposta do SVH para estímulos radiais e angulares acoplados. Acreditamos que estímulos elementares radiais e angulares simples ou acoplados são importantes para o processamento visual de objetos simétricos complexos, por exemplo, faces. Estes também podem ser importantes para medir a sensibilidade de células em áreas do córtex visual sensíveis a estímulos simétricos.

\section{Método}

\section{Participantes}

Participaram dos experimentos 6 voluntários de ambos os sexos com acuidade visual normal ou corrigida na faixa etária de 19-26 anos.

\section{Equipamentos e Estímulos}

Foi utilizado o sistema desenvolvido no próprio laboratório, que compreende um monitor de vídeo SONY-BVM-1910, com entrada "RGBsync" entrelaçada e interfaciado a um microcomputador 486 de $30 \mathrm{MHz}$ através de um placa de aquisição Data-Translation DT-2853. Um programa escrito em linguagem "C" foi desenvolvido para executar os experimentos. Uma cadeira giratória foi fixada a $150 \mathrm{~cm}$ da tela do monitor de vídeo. Uma tábua cinza foi situada acima do monitor de vídeo para onde o voluntário, entre uma apresentação e outra, deveria fixar o olhar com o objetivo de diminuir os efeitos de pósimagem. A luminância média foi de $6,9 \mathrm{~cd} / \mathrm{m}^{2}$ ajustada por um fotômetro do tipo SPOT METTER, com precisão de um grau, ASAHI PENTAX. A luminância mínima foi de $6,2 \mathrm{~cd} / \mathrm{m}^{2}$ e a máxima de $7,5 \mathrm{~cd} / \mathrm{m}^{2}$. O ambiente do laboratório era cinza para melhor controle da luminância.

Os estímulos foram gerados em tons de cinza e apresentados em tempo real no monitor. Os estímulos de freqüências espaciais (grades senoidais), freqüências radiais e freqüências radiais/ angulares acoplados utilizados foram 0,$2 ; 0,3 ; 0,5 ; 0,8 ; 1 ; 2 ; 3 ; 4 ; 5$; 6 e 9 ciclos por grau de ângulo visual (cpg). O estímulo de teste foi qualquer uma das freqüências acima, exceto para os estímulos de testes acoplados, para os quais as freqüências radiais acima foram multiplicadas ponto por ponto a freqüência angular de 4 ciclos $/ 360^{\circ}$. O estímulo de fundo foi sempre um padrão homogêneo com luminância média de $6,9 \mathrm{~cd} / \mathrm{m}^{2}$. Todos os estímulos eram circulares, gerados em tons de cinza, com um diâmetro de 7,25 graus de ângulo visual a $150 \mathrm{~cm}$ de distância da tela (Figura 1).
A Figura 1 ilustra exemplos de freqüências espaciais, radiais e radiais/angulares acopladas. Mais informações sobre estes estímulos (Ex.: equações matemáticas), podem ser encontrados na literatura (Santos \& Simas, 2001b; Simas \& Santos, 2002a, 2002b; Simas, Santos \& Thiers, 1997).
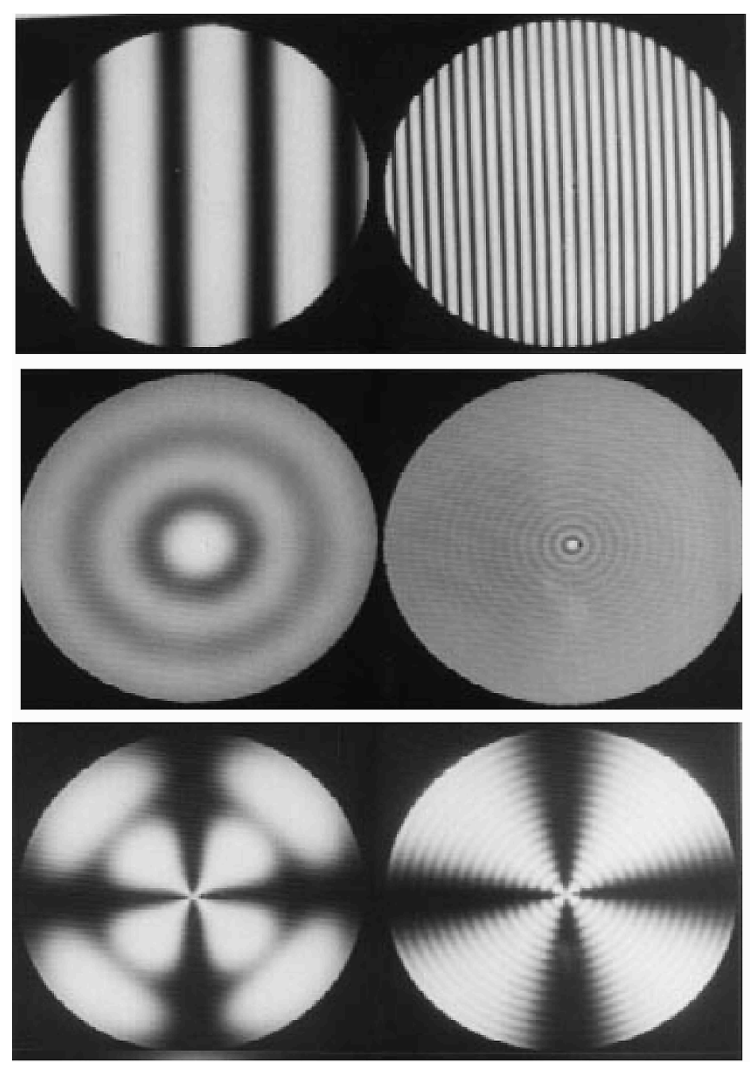

Figura 1. Exemplos de estímulos elementares de 0,5 e 3 cpg. Acima, grades senoidais; no centro, freqüências radiais e embaixo, freqüências radiais de 0,5 e 3 cpg acopladas à freqüência angular de 4 ciclos $/ 360^{\circ}$.

\section{Procedimento}

As estimativas foram realizadas pelo método da escolha forçada (Santos, 1999; Simas \& cols., 1992, 1997; Simas \& Santos, 2002a, 2002b). Este método foi baseado no estudo de Wetherill \& Levitt (1965), que calcula a probabilidade de acertos consecutivos por parte do voluntário, ou seja, em cerca de 100-150 apresentações de escolhas entre os dois estímulos, o estímulo de teste é percebido $79 \%$ das vezes pelo voluntário. $\mathrm{O}$ procedimento para medir o limiar para cada freqüência consistiu na apresentação sucessiva simples do par de estímulos e o voluntário teria que escolher dentre eles qual continha a freqüência de teste (espacial, radial ou radial/angular acoplada). O critério adotado para variar o contraste da freqüência de teste era o de três acertos consecutivos para decrescer uma unidade e um erro para acrescer da mesma unidade $(0,08 \%)$. 
Durante cada sessão experimental era apresentada uma seqüência de estímulos que foi iniciada por um sinal sonoro seguido imediatamente pela apresentação do primeiro estímulo por $2 \mathrm{~s}$, seguido de um intervalo entre estímulos de $1 \mathrm{~s}$, seguido pela apresentação do segundo estímulo por $2 \mathrm{~s}$ e da resposta do voluntário. A ordem de apresentação dos estímulos era aleatória. Se a resposta do voluntário fosse correta, era seguida por outro sinal sonoro e um intervalo de $3 \mathrm{~s}$ para a seqüência se repetir. Em outras palavras, o intervalo entre tentativas era de $3 \mathrm{~s}$ independente da resposta (ou escolha) ser correta ou não. $\mathrm{O}$ sinal sonoro que indicava o início da apresentação do par de estímulos e o que indicava a escolha correta eram diferentes e discretos. A sessão experimental variava em duração dependendo dos erros e acertos do voluntário até proporcionarem um total de 10 máximos e 10 mínimos conforme requerido para o final automático da mesma.

Cada um dos pontos (ou freqüências) da curva de limiar de contraste para freqüências radiais ou freqüências radiais/angulares acopladas corresponde a uma sessão experimental. Cada freqüência foi estimada pelo menos duas vezes, em dias diferentes, por cada um dos seis voluntários. Em média, 12 curvas foram medidas para cada conjunto de estímulo (espaciais, radiais e radiais/ angulares acoplados) gerando uma amostra de aproximadamente 240 valores para cada um dos pontos estimados. Todas as estimativas foram medidas à distância de $150 \mathrm{~cm}$, com visão binocular.

Os voluntários foram orientados antes da sessão a pressionar a barra de espaço quando julgassem que o estímulo de teste tivesse sido apresentado primeiro e qualquer tecla acima da barra de espaço quando julgasse que o mesmo tivesse sido apresentado em segundo lugar, isto é, após o estímulo de fundo. Em outras palavras, a tarefa do observador foi escolher sempre o estímulo que continha a freqüência espacial, radial ou radial/ angular acoplada.

\section{Resultados}

A Figura 2 mostra as curvas de limiar de contraste para os estímulos de freqüências espaciais (grades senoidais, 1/CSF), radiais $(1 / \mathrm{rCSF})$ e radiais/angulares acoplados $(1 / \mathrm{raCSF})$. As freqüências senoidais, radiais e radiais/angulares acopladas são apresentadas nos gráficos em função da quantidade de contraste ou da sensibilidade ao contraste do SVH.

Nosso tratamento estatístico foi estimar o erro-padrão da média para cada distribuição de 240 valores mensurados para cada ponto e corrigidos para o tamanho da amostra pelo estatístico t-Student para obter o nível de confiança de 99\%. Em experimentos anteriores, foi estabelecido que o erro-padrão da média, corrigido pelo tamanho da amostra representando intervalo de confiança de $99 \%$ pelo estatístico $t$-Student, de acordo com o número de valores mensurados, é um critério

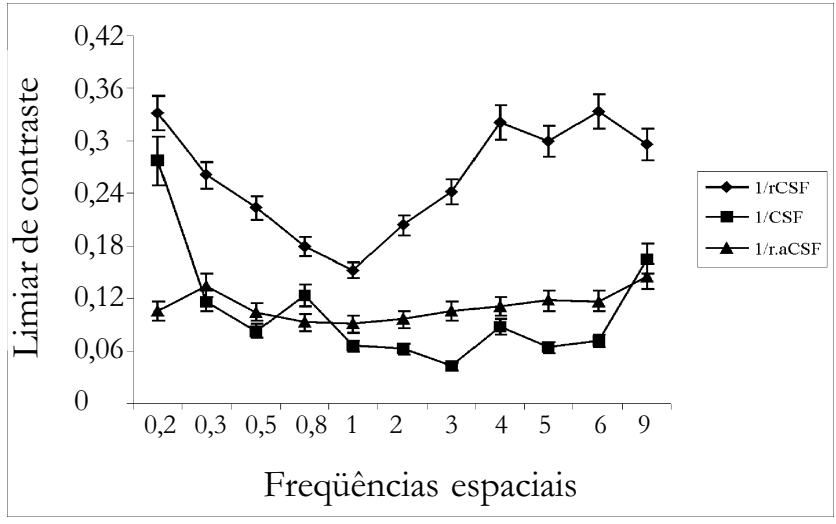

Figura 2. Curvas de limiar de contraste para freqüências radiais $(1 / \mathrm{rCSF})$, grades senoidais $(1 / \mathrm{CSF})$ e freqüências radiais acopladas à freqüência angular de 4 ciclos $/ 360^{\circ}$ $(1 / \mathrm{r} . \mathrm{aCSF})$. As barras verticais correspondem ao erropadrão da média corrigido para o tamanho da amostra pelo t-Student com intervalo de confiança de 0,99 .

mais estrito do que a utilização da ANOVA ou teste t para amostras correlacionadas. Por exemplo, quando os intervalos dos erros-padrão da média assim corrigidos se superpõem até mesmo pela metade, ainda assim um teste $t$-Student para amostras correlacionadas revela diferenças entre as médias que são significativas $\operatorname{com} p<0,05$. Nos casos em que os erros barras não se sobrepõem, as médias são significativas $\operatorname{com} p<0,001$. A ANOVA tende a mostrar interações e efeitos significativos em todos fatores e não acrescenta muita informação.

A Figura 2 mostra a média geral dos limiares de contraste para todos os participantes em função das freqüências senoidais $(1 / \mathrm{CSF})$, freqüências radiais $(1 / \mathrm{rCSF})$ e freqüências radiais/angulares acopladas ( $1 / \mathrm{r}$.aCSF) em ciclos por grau de ângulo visual (cpg). As barras verticais na Figura 2 indicam os erros-padrão das médias com intervalos de $99 \%$ de confiança corrigidos para o tamanho da amostra pelo estatístico t-Student.

A função de sensibilidade ao contraste (CSF) é o inverso da curva de limiar de contraste (1/CSF). Em outras palavras, quanto menor o limiar de contraste maior a sensibilidade do sistema visual humano e vice-versa. Assim, os menores valores de limiares correspondem aos maiores valores de sensibilidade ao contraste.

Observa-se que a sensibilidade ao contraste e a faixa de maior sensibilidade variaram de acordo com o tipo de estímulo (Figura 2). Por exemplo, a faixa de maior sensibilidade ficou em $1 \mathrm{cpg}$ para estímulos radiais, $\mathrm{J}_{0}$, (1/ rCSF), em 3 cpg para freqüências espaciais (1/CSF) e entre 0,8-1 cpg para os estímulos radiais acoplados à freqüência angular de 4 ciclos $(1 / \mathrm{r}$.aCSF $)$. 
Os resultados demonstram, ainda, que o SVH apresenta maior sensibilidade para freqüências espaciais e menor sensibilidade para freqüências radiais. $\mathrm{O}$ acoplamento de freqüências radiais à angular de 4 ciclos $(1 / \mathrm{r}$.aCSF $)$ proporcionou um aumento na sensibilidade ao contraste da ordem de pelo menos 1,6 vezes em favor dos estímulos acoplados quando comparados à $1 / \mathrm{rCSF}$ (Figura 2). A ordem crescente dos estímulos do menos sensível para o mais sensível foi $1 / \mathrm{rCSF}<1 / \mathrm{r}$.aCSF $<1 / \mathrm{CSF}$.

Estes resultados são consistentes com a existência de mecanismo sensíveis a estímulos circularmente simétricos ou estímulos em coordenadas polares simples ou acoplados.

\section{Discussão}

Os resultados mostram que a curva de limiar para estímulos circularmente simétricos radiais e radiais/angulares acoplados em coordenadas polares $(1 / \mathrm{rCSFe} 1 / \mathrm{raCSF})$ possuem as mesmas características gerais que a curva de limiar para estímulos em coordenadas cartesianas do tipo grade senoidal, 1/CSF (Figura 2). Isto é, faixa de máxima sensibilidade nas freqüências intermediárias, aproximadamente no centro da curva, com atenuações nas freqüências baixas e altas, extremos da curva. Teoricamente, a 1/rCSF (ou 1/raCSF) para estímulos radiais e radiais/angulares acoplados em coordenadas polares seriam tão importantes quanto a 1/CSF paragrades senoidais em coordenadas cartesianas (Santos, 1999; Simas \& Dodwell, 1990; Simas \& cols., 1992, 1997; Simas \& Santos, 2002a, 2002b), podendo até ser mais relevantes, dependendo dos atributos visuais que se pretende estudar, como por exemplo, a percepção de imagem simétrica (Ex.: faces) ou outros atributos tais como anisotropia, fase e análise espacial em coordenadas polares. Inclusive, ao observar os limiares de contraste do SVH para estímulos radiais, radiais/ angulares acoplados e grade senoidal (Figura 2), parece que cada conjunto de estímulo tem atributos peculiares relacionados à deteç̧ão da forma e do contraste que os diferenciam entre si. As alterações na sensibilidade do SVH para esses estímulos são dificeis de ser explicadas por um mecanismo único, conforme veremos a seguir.

\section{Sensibilidade para estímulos radiais versus grades senoidais}

O fato de o SVH ser mais sensível para grades senoidais do que para estímulos radiais modulados pela função cilíndrica (Figura 2) de Bessel $\left(J_{0}\right)$ já era esperado, pois isto já havia sido demonstrado por Kelly (Kelly, 1982; Kelly \& Magnuski, 1975). Esta redução de sensibilidade do SVH para estímulos radiais com contraste modulado pela função esférica (Simas \& Santos, 2002b) e pela função cilíndrica de Bessel (presente estudo) pode estar relacionada à própria definição matemática do estímulo que varia de acordo com a função esférica ou cilíndrica de Bessel. Os estímulos radiais apresentam contraste mais intenso no centro e menos intenso na periferia (Figura 1, ver também Simas \& Santos, 2002b) tendendo a ser constante no infinito. Variando a ordem $(n)$ da função de Bessel esférica $\left(j_{11}\right)$ ou cilíndrica $\left(J_{12}\right)$, a amplitude do contraste próxima ao centro diminui ao mesmo tempo que o anel central tende a se expandir (Simas \& Santos, 2002b).

A redução na sensibilidade ao contraste para estímulos radiais $(1 / \mathrm{rCSF})$ comparados a grades senoidais $(1 / \mathrm{CSF})$ pode ser devido aos estímulos radiais não possuírem amplitude equivalente ao longo do estímulo, isto é, eles apresentam amplitude máxima no centro (fóvea) e vai diminuindo para periferia do campo visual (Simas \& Santos, 2002b). Ao contrário, a amplitude da grade senoidal é equivalente ao longo de todo o estímulo ou campo visual. Então, é possível que a quantidade de canais ou a forma (intensidade) com que os canais específicos para cada padrão são ativados (na fóvea e próximo à fóvea) façam a diferença. A relação lógica por trás desse processo é que, se os dois padrões ativam canais específicos de formas diferentes, aquele que ativa mais intensamente o maior número de canais poderia apresentar maior sensibilidade. Esta hipótese pode ser reforçada por estudos neurofisiológicos que sugerem que estímulos em coordenadas cartesianas e polares são processados por mecanismos distintos (Desimone \& Schein, 1987; Gallant \& cols., 1993, 1996; Kobatake \& Tanaka, 1994; Tanaka, 1996; Van Essen, Anderson \& Felleman, 1992; Wilson \& Wilkinson, 1998; Wilson \& cols., 1997).

\section{Sensibilidade para estímulos radiais versus radiais/ angulares acoplados}

Simas e colaboradores demonstraram que a sensibilidade ao contraste para freqüências angulares $(1 / \mathrm{aCSF})$ é muito mais alta do que para freqüências radiais (Simas \& cols., 1997; Simas \& Santos, 2002b). Neste sentido, esperávamos que o acoplamento de freqüências radiais a angulares melhorasse a sensibilidade ao contraste do SVH. Isto realmente ocorreu e pode ser observado na Figura 2. O aumento da sensibilidade a favor de freqüências radiais acopladas à freqüência angular de 4 ciclos $/ 360^{\circ}$ em relação à freqüência radial simples, inicialmente, nos leva a hipótese de que estímulos radiais/ angulares acoplados podem ativar canais para freqüências radiais e canais para freqüências angulares com orientações diferentes (verticais e horizontais), cujos efeitos se somam espacialmente aumentando a sensibilidade do SVH. Vincent e Regan (1995) demonstraram que o processamento de orientação, freqüência espacial e contraste são codificados independentemente e paralelamente, sendo que a performance doSV melhora quando esses atributos são processados simultaneamente em paralelo. Se levarmos em conta que os estímulos radiais são circularmente 
simétricos e sofrem pouca influência da orientação, pode-se esperar menor sensibilidade para estímulos radiais puros. Esta hipótese é reforçada por Simas e colaboradores que mostram maior sensibilidade para freqüências angulares comparadas a freqüências espaciais e radiais. Estudos neurofisiológicos, também, sugerem que estímulos em coordenadas polares são processados por mecanismos distintos e em áreas corticais distintas (Desimone \& Schein, 1987; Gallant \& cols., 1993, 1996; Kobatake \& Tanaka, 1994; Tanaka, 1996; Van Essen \& cols., 1992). Por exemplo, em 1994 Kobataka e Tanaka encontraram células seletivas a estímulos radiais na área visual V2, enquanto Gallant e colaboradores (1993, 1996) encontraram evidências para estímulos radiais e angulares na área visual V4.

Os dados discutidos até aqui demonstram que a sensibilidade do SVH para determinados estímulos depende das características espaciais, físicas, do modelo matemático e do sistema de coordenadas (polares ou cartesianas) que definem os mesmos. Estas características, dentre outras, por sua vez, estão relacionadas à detecção, ao processamento neural e à integração final da imagem percebida. Levando em conta estas ou partes destas considerações, além do contexto experimental em que os estímulos estão inseridos, podemos afirmar que o SVH é mais sensível para freqüências angulares e menos sensíveis para freqüências radiais puras. Assim, a ordem decrescente do mais sensível para o menos sensível neste trabalho foi: $1 / \mathrm{CSF}>1 / \mathrm{raCSF}>1 / \mathrm{rCSF}$.

Assim podemos concluir que o SVH é sensível a estímulos descritos em um sistema de coordenadas polares. Estes achados reforçam a idéia de um possível envolvimento de padrões radiais e angulares em coordenadas polares no processamento e reconhecimento de objetos.

Estes resultados estão de acordo com dados psicofísicos (Wilson \& Wilkinson, 1998; Wilson \& cols, 1997) e fisiológicos que relatam o envolvimento de V4 e IT no processamento e amostragem de áreas grandes do campo visual ou processamento de forma global, por exemplo faces (Bruce, Desimone \& Gross, 1981; Desimone, 1991; Desimone \& Schein, 1987; Gallant \& cols., 1993, 1996; Heywood, Gadotti \& Cowey, 1992; Kobatake \& Tanaka, 1994; Merigan, 1996; Tanaka, 1996). A seguir tentaremos resumir algumas evidências neurofísiológicas favoráveis ao processamento de estímulos em coordenadas polares.

Evidências neurofisiologicaspara processamento de estímulos espaciais em coordenadas polares: existem fortes evidências, baseadas em estudos psicofísicos e neurofisiológicos, de que o processamento linear ou filtragem espacial local sintonizada para orientação e freqüência espacial de estímulos projetados na retina ocorre nos estágios iniciais da visão, isto é, em neurônios do córtex visual primário, V1 (R. L. De Valois \&
K. K. De Valois, 1988; Manahilov \& Simpson, 2001; Wilkinson \& cols., 2000; Wilson \& Wilkinson, 1998; Wilson \& cols., 1997). Por outro lado, até o momento não existe nenhum relato de que unidades neurais da área visual primária, V1, executem filtragem de estímulos em coordenadas polares, amostrando áreas grandes do campo visual. Por outro lado, existem dados baseados em estudos psicofísicos em humanos (Wilkinson \& cols., 1998; Wilson \& Wilkinson, 1997, 1998; Wilson \& cols, 1997), neurofisiológicos em primatas (Bruce \& cols., 1981; Desimone, 1991; Desimone \& Schein, 1987; Gallant \& cols., 1993, 1996; Hegde \& Van Essen, 2000; Heywood \& cols., 1992, Merigan, 1996; Van Essen \& cols., 1992; Young, 1992) e com imagem de ressonância magnética funcional em humanos (Wilkinson \& cols., 2000), de que o processamento da informação que ocorre em vias intermediárias (Ex.: V4) e áreas mais avançadas (Ex.: IT) do sistema visual são modeladas por filtros espaciais globais organizadas em coordenadas polares. Alguns desses trabalhos citados acima destacaram que a área V4 pode formar o principal estágio intermediário da visão de forma de V1 para IT (Heywood \& cols., 1992, Merigan, 1996; Van Essen \& cols., 1992; Wilkinson \& cols., 2000; Young, 1992). Inclusive, alguns estudos mostraram que os campos receptivos de neurônios em V4 são muito maiores do que os campos receptivos de neurônios de V1 (Desimone \& Schein, 1987; Kobatake \& Tanaka, 1994; Merigan, 1996; Tanaka, 1996). Assim, é possível que o processamento e integração de padrões em coordenadas polares ocorram em áreas visuais extra-estriatais (Ex.: V4 e IT). O estudo de Wilkinson e colaboradores (2000) com a técnica de imagem de ressonância magnética funcional em humanos reforçou os estudos psicofísicos com humanos e neurofisiológicos com primatas que demonstraram inicialmente que estímulos em coordenadas polares são processados em áreas extra-estriatais (Ex.: V4 e IT). Além disto, este estudo reforçou a idéia de que o sistema visual de humanos e de primatas são muito semelhantes. De mesma forma, em artigo recente, Connor (2000) afirmou que macacos percebem objetos de maneira semelhante a humanos.

A idéia geral que fica, baseada nos nossos dados e nos dados encontrados na literatura, é que se não existem mecanismos distintos seletivos a freqüências angulares, radiais, radiais/angulares acopladas e grade senoidal, pelo menos devem existir mecanismos distintos ou áreas distintas para estímulos em coordenadas polares e para estímulos em coordenadas cartesianas.

Todos os resultados apresentados e discutidos neste trabalho são consistentes com a possibilidade de que estímulos em coordenadas polares são importantes para caracterizar a resposta do SVH à forma. Estes resultados integram um conjunto de dados que vem sendo formulado 
sobre processamento de estímulos elementares em coordenadas polares e o processamento e o reconhecimento de faces com enfoque no processamento de áreas grandes do campo visual ou processamento global.

Em síntese, estes resultados fornecem evidências para mecanismos distintos sintonizados para percepção de padrões simétricos em coordenadas polares. Por outro lado, estes resultados sobre detecção por si só não podem provar que o SV utiliza freqüências radiais e angulares simples ou acopladas para processar e reconhecer objetos ou uma cena visual. Portanto, o fato de o SVH ser sensível a estímulos desta natureza é um indício forte da importância destes estímulos no processamento visual da forma, mas não que estes sejam realmente empregados neste processo.

\section{Referências}

Amidor, I. (1997). Fourier spectrum of radially periodic images. Journal of the Optical Society of America A, 14, 816-826.

Blakemore, C. \& Campbell, F. C. (1969a). Adaptation to spatial stimuli. Journal of Physiology, 200, 11-12.

Blakemore, C. \& Campbell, F. C. (1969b). On the existence of neurons in the human visual system selectively sensitive to the orientation and size of retinal images. Journal of Physiology, 203, 237-260.

Blakemore, C., Nachmias, J. \& Sutton, P. (1970). The perceived spatial frequency selective neurones in the human brain. Journal of Physiology, 210, 727-750.

Braddick, O., Campbell, F. W. \& Atkinson, J. (1978). Channels in vision: Basic aspects. Em R. Held, H. W. Leibowitz \& H. L. Teuber (Orgs.), Handbook of Sensory physiology: Perception. (Vol. V, pp. 3-38). New York: Springer-Verlag.

Bruce, C. J., Desimone, R. \& Gross, C. G. (1981). Visual properties of neurons in a polysensory area in superior temporal sulcus of the macaque. Journal of Neurophysiology, 46, 369-384.

Campbell, F. W. \& Maffei, L. (1970). Electrophysiological evidence for the existence of orientation and size detectors in the human visual system. Journal of Physiology, 207, 635-652.

Campbell, F. W. \& Maffei, L. (1974). Contrast and spatial frequency. Scientific American, 231, 106-114.

Campbell, F. W. \& Robson, F. G. (1968). Application of the Fourier analysis to the visibility of gratings. Journal of Physiology, 197, 551-566.

Connor, C. E. (2000). Visual perception: Monkeys see things our way. Current Biology, 10, R836-R838.

Cornsweet, T. N. (1970). Vision perception. New York: Academic Press.

De Valois, R. L. \& De Valois, K. K. (1988). Spatial vision. New York: Oxford University Press.

Desimone, R. (1991). Face-selective cells in the temporal cortex of monkeys. Journal of Cognitive Neuroscience, 3, 1-8.

Desimone, R. \& Schein, S. J. (1987). Visual properties of neurons in area V4 of macaque: Sensitivity to stimulus form. Journal of Neurophysiology, 57, 835-867.

Gallant, J. L., Brau, J. \& van Essen, D. C. (1993). Selectivity for polar hyperbolic, and cartesian gratings in macaque visual cortex. Science, 259, 100-103.

Gallant, J. L., Connor, C. E., Rakshit, S., Lewis, J. W. \& Van Essen, D. C. (1996). Neural responses to polar, hyperbolic, and cartesian gratings in area $\mathrm{V} 4$ of the macaque monkey. Journal of Neurophysiology, 76, 2718-2739.

Graham, N. (1972). Spatial frequency channels in the human visual system: Effects of luminance and pattern drift rate. Vision Research, 12, 53-63.

Graham, N. \& Nachmias, J. (1971). Detection of grating patterns containing two spatial frequencies: A comparison of single-channel and multiple channel models. Vision Research, 11, 251-259.
Hegde, J. \& Van Essen, D. C. (2000). Selectivity for complex shapes in primate visual area V2. Journal of Neuroscience, 20, RC61.

Hess, R. F., Wang, Y. -Z., Demanins, R., Wilkinson, F. \& Wilson, H. R. (1999). A deficit in strabismic amblyopia for global shape detection. Vision Research, 39, 901-914.

Heywood, C. A., Gadotti, A. \& Cowey, A. (1992). Cortical area V4 and its role in the perception of color. Journal of Neuroscience, 12, 4056-4065.

Kelly, D. H. (1960). J stimulus patterns for vision research. Journal of the Optical Society of America, 50, 1115-1116.

Kelly, D. H. (1982). Motion and vision: IV. Isotropic and anisotropic spatial response. Journal of the Optical Society of America, 72, 432-439.

Kelly, D. H. \& Magnuski, H. S. (1975). Pattern detection and the two dimensional Fourier transform: Circular targets. Vision Research, 15, 911-915.

Kobatake, E. \& Tanaka, K. (1994). Neuronal selectivities to complex object features in the ventral visual pathway of the macaque cerebral cortex. Journal of Neurophysiology, 71(3), 856-867.

Maffei, L. \& Fiorentini, A. (1973). The visual cortex as a spatial frequency analyzer. Vision Research, 13, 1255-1267.

Manahilov, V. \& Simpson, W. A. (2001). Energy model for contrast detection: Spatial-frequency and orientation selectivity in grating summation. Vision Research, 41, 1547-1560.

Merigan, W. H. (1996). Basic visual capabilities and shape discrimination after lesions of extrastriate area V4 in macaques. Visual Neuroscience, 13, 51-60.

Mortensen, U. \& Meinhardt, G. (2001). Detection of simple radially symmetric targets: Further evidence for the matched filter processing scheme in human pattern detection. Biological Cybernetics, 84, 63-74.

Pantle, A. \& Sekuler, R. (1968). Size detecting mechanisms in human vision. Science, 162, 1146-1148.

Sachs, M. B., Nachmias, J. \& Robson, J. G. (1971). Spatial frequency channels in human vision. Journal of the Optical Society of America, 61, 1176-1186.

Santos, N. A. (1999). Sistema visual humano: Curvas de sensibilidade e filtragem de frequiências angulares, radiais e radial/ angulares acopladas. Tese de Doutorado não-publicada, Curso de Pós-Graduação em Neurociências e Comportamento, Instituto de Psicologia, Universidade de São Paulo. São Paulo, Brasil.

Santos, N. A. \& Simas, M. L. B. (2001a). Percepção e processamento visual da forma: Discutindo modelos teóricos atuais. Psicologia: Reflexão \& Crítica, 14, 151-160.

Santos, N. A. \& Simas, M. L. B. (2001b). Função de sensibilidade ao contraste: Indicador da percepção visual da forma e da resolução espacial. Psicologia: Reflexão e Crítica, 14, 589-597.

Santos, N. A. \& Simas, M. L. B. (2002). Percepção e processamento visual da forma em humanos: Filtros de freqüências radiais de 1 e 4 cpg. Psicologia: Reflexão e Crítica, 15, 383-391.

Simas, M. L. B. (1985). Linearity and domain invariance in the visual system. Ph.D. thesis. Queen's University at Kingston, Ontario, Canada: University Microfilms International. Ann Arbor: Michigan.

Simas, M. L. B. \& Dodwell, P. C. (1990). Angular frequency filtering: A basis for pattern decomposition. Spatial Vision, 5, 59-74.

Simas, M. L. B., Frutuoso, J. T. \& Vieira, F. M. (1992). Inhibitory sidebands in multiple angular filters in the human visual system. Brazilian Journal of Medical and Biologocal Research, 25, 919-923.

Simas, M. L. S. \& Santos, N. A. (2002a). Narrow-band 1, 2, 3, 4, 8, 16 and 24 cycles/ $360^{\circ}$ angular frequency filters. Brazilian Journal of Medical and Biological Research, 35, 243-253.

Simas, M. L. S. \& Santos, N. A. (2002b). Contrast sensitivity to radial frequencies modulated by Jn and jn Bessel profiles. Brazilian Journal of Medical and Biological Research, 35, 1357-1366.

Simas, M. L. S., Santos, N. A. \& Thiers, F. A. (1997). Contrast sensitivity to angular frequency stimuli is higher than that for sine wave gratings in the respective middle range. Brazilian Journal of Medical and Biological Research, 30, 633-636.

Tanaka, K. (1996). Inferotemporal cortex and object vision. Annual Review of Neuroscience, 19, 109-139. 
Van Essen, D. C., Anderson, C. H. \& Felleman, D. J. (1992). Information processing in the primate visual system: An integrated systems perspective. Science, 255, 419-423.

Verrall, S. C. \& Kakarala, R. (1998). Disk-harmonic coefficients for invariant pattern recognition. Journal of the Optical Society of America A, 15, 389-401.

Vincent, A. \& Regan, D. (1995). Parallel independent encoding of orientation, spatial frequency, and contrast. Perception, 24, 291-299.

Wetherill, G. B. \& Levitt, H. (1965). Sequential estimation of points on a psychometric function. The British Journal of Mathematical and Statistical Psychology, 48, 1-10.

Wilkinson, F., Wilson, H. R. \& Habak, C. (1998). Detection and recognition of radial frequency patterns. Vision Research, 38, 3555-3568.

Wilkinson, F., James, T. W., Wilson, H. R., Gati, J. S., Menon, E. S. \& Goodale, M. A. (2000). An fMRI study of the selective activation of human extrastriate form vision areas by radial and concentric gratings. Current Biology, 10, 14551458.

Wilson, H. R., Levi, D., Maffei, L., Rovamo, J. \& De Valois, R. (1990). The perception of form: Retina to striate cortex. Em S. W. Spillmann \& J. S. Werner (Orgs.), Visual perception: The neurophysiological foundation (pp. 231-271). New York: Academic Press.
Wilson, H. R. \& Wilkinson, F. (1997). Evolving concepts of spatial channels in vision: From independence to nonlinear interactions. Perception, 26, 939-960.

Wilson, H. R. \& Wilkinson, F. (1998). Detection of global structure in glass patterns: Implications for form vision. Vision Research, 38, 2933-2947.

Wilson, R. W., Wilkinson, F. \& Asaad, W. (1997). Concentric orientation summation in human form vision. Vision Research, 37, 2325-2330.

Young, M. P. (1992). Objective analysis of topological organization of the primate cortical visual system. Nature, 358, 152-155.

\section{Sobre os Autores}

Natanael Antonio dos Santos é Doutor em Psicologia (Neurociências e Comportamento) pelo Instituto de Psicologia Experimental da USP (1999). Professor Adjunto do Departamento de Psicologia da Universidade Federal da Paraíba. É Coordenador do Laboratório de Processamento Visual - LabPVis. Linha de Pesquisa: Percepção e Processamento Visual da Forma em Humanos.

Maria Lúcia de Bustamante Simas é Doutora em Psicologia pela Queen's University, Ontario, Canada (1985). Professora Adjunta do Departamento de Psicologia da Universidade Federal de Pernambuco. É Coordenadora do Laboratório de Percepção Visual - LabVis. Linha de Pesquisa: Percepção e Processamento Visual da Forma. Renata Maria Toscano Barreto Lyra Nogueira é Estudante de Psicologia da Universidade Federal de Pernambuco. Bolsista de Iniciação Científica CNPq, vinculada ao LabVis-UFPE.

Recebido: $23 / 05 / 2003$

$1^{a}$ Revisão: $28 / 08 / 2003$ Aceite Final: 09/10/2003 